\title{
Nanosecond Pulsed Electric Fields: A New Stimulus to Activate Intracellular Signaling
}

\author{
Stephen J. Beebe and Karl H. Schoenbach \\ Frank Reidy Research Center for Bioelectrics, Eastern Virginia Medical School and Old Dominion University, Norfolk, VA 23510, USA
}

\begin{abstract}
When new technologies are introduced into the scientific community, controversy is expected and both excitement and disappointment enrich the lives of those who initiate the new ideas. It becomes the mission of the "inventors" to embrace the burden of proof to establish their ideas and convince the skeptics and disbelievers who will undoubtedly temper their enthusiasm and test their patience. While open mindedness is generally a scientific motto, those who review patents, manuscripts, and grants do not always readily practice it, even when the evidence is convincingly presented; old ideas and concepts often die hard. So it has been and still is in many instances as engineers, physicists, biologists, and physicians pursue innovative ideas and novel technolo-
\end{abstract} gies.

So what is "Bioelectrics"? It is the application of ultrashort pulsed electric fields to biological cells, tissues, and organs. More specifically, it is the analysis of how these biological systems respond to high electric fields (10-100 s of $\mathrm{kV} / \mathrm{cm}$ ) when applied with nanosecond (1-300) durations. Compressing electrical energy by means of pulsed power techniques allows the generation of ultrashort (billionth of a second) electrical pulses [1]. Because the pulses are so short the energy density is quite low and therefore nonthermal. However, the power is extremely high generating billions of watts. This can be compared to a coal power plant, which generates less than billion watts, but does it continuously. For example, for a $10 \mathrm{~ns}, 40 \mathrm{kV}$, $10 \Omega$ pulse generator, the power provided by the pulse is $160 \mathrm{MW}$, however, the energy is only 1.6 J. Depositing this

Correspondence and reprint requests to Stephen J. Beebe, Frank Reidy Research Center for Bioelectrics, Eastern Virginia Medical School and Old Dominion University, Norfolk, VA 23510, USA, E-mail: beebesj@evms.edu

This is an open access article distributed under the Creative Commons Attribution License which permits unrestricted use, distribution, and reproduction in any medium, provided the original work is properly cited. energy into one milliliter of water causes an increase in temperature by just one third of one degree Celsius. We have referred to these pulses as ultrashort, high-voltage pulsed electric fields or nanosecond pulsed electric fields (nsPEFs). These conditions are most certainly unique and do not exist in nature. Thus, this provides an opportunity to determine how cells respond to stimuli that they have not evolved to recognize. Undoubtedly, cells respond to nsPEFs in diverse and cell-type-specific ways. This suggests that nsPEFs represent a distinctive, non-ligand stimulus that can disclose basic cell-type-specific differences for responses to the external environment and can also be investigated for potential therapeutic and/or diagnostic applications. A patent has been issued and several provisional patents have been filed for devices and applications of nsPEFs to cells and tissues for a wide range of applications.

The use of electric fields on biological systems is not new, but it has been a common misconception that nsPEFs are also not new. A method called electroporation has been used for decades to introduce drugs and/or DNA into cells for basic science or for therapeutic purposes. These electric fields charge the plasma membrane causing the temporary formation of "pores" or breaching of plasma membrane integrity that allows the entry of "foreign" molecules into the cell interior. However, compared to nsPEFs classical plasma membrane electroporation pulses are relatively long (microseconds to milliseconds) and with lower electric fields $(\leq 1 \mathrm{kV} / \mathrm{cm})$. Thus, nsPEFs can be orders of magnitude shorter in duration and higher in electric field. For example, during a $1 \mathrm{~ms}$ electroporation pulse light travels 982,000 feet (186 miles). During a $10 \mathrm{~ns}$ pulse, light travels about 10 feet. Nevertheless, because nsPEF applications are an extension of classical plasma membrane electroporation, the effects of nsPEFs are often confused with effects of electroporation on the plasma membrane.

Not so! Especially as the pulse duration is decreased below the charging time of the plasma membrane. The exclamation point serves a special note that cell response 
phenomena have now significantly changed as the risetime and the pulse duration are below times required to fully charge the plasma membrane. As opposed to responses to classical plasma membrane electroporation, nsPEF affect intracellular structures (membranes) and functions (cell signaling), which may or may not involve measurable responses from the outer plasma membrane. This primarily depends on the pulse duration, pulse rise time, and electric field. nsPEFs enter new biological and cellular vistas with dimensions, dynamics, and kinetics focused more on intracellular mechanisms $[2,3]$. Nevertheless, nsPEFs have effects on the plasma membrane that are direct electric field, nonbiological effects, as well as secondary biological effects. While biological effects on the plasma membrane, such as phosphatidylserine (PS) externalization associated with apoptosis are readily measured, they can be confused with PS externalization resulting from direct electric field effects. Direct electric field effects on plasma membrane integrity are often harder to determine because they occur at levels that are often below the level of detection by fluorophores and/or molecular probes are too large for small pores, referred to as nanopores, which are believed to be present with nsPEFs. Such nanopores and nanochannels for phosphatidylserine externalization are predicted based on modeling and simulation studies [4]. What effects nsPEFs might have on proteins and ion channels are not yet investigated. How diminishing effects on plasma membrane structures and functions interface with increasing effects on intracellular structures and functions as the pulse duration decreases remain the basis for continued research in bioelectrics.

we have referred to the nsPEF-induced occurrence of greater effects on intracellular membranes and lesser effects on plasma membranes as intracellular electromanipulation (IEM). We used the term "manipulation" instead of "electroporation" because it is yet to be determined whether nsPEF-induced effects on intracellular structures are similar to classical electroporation on the plasma membrane. Since nsPEFs applications are an extension of classical plasma membrane electroporation, it is reasonable and prudent to consider membrane charging as a mechanism for nsPEF effects. While the membranes may not be fully charged for a $10 \mathrm{~ns}$ pulse, they are charging during the pulse. However, as the pulse duration decreases to and below 1 ns, charging may not be a major factor. Here pure physics meets biology head on and new dimensions and other mechanisms may be encountered.

This introduction to nsPEFs provides all of the superlatives and fervor that could be expected from physicists and biologists with a brand new toy. So what data support the unique effects of nsPEFs on biological systems? Most of the work has been done on cells in cultures, however an increasing number of studies are being conducted on tissues, including fibrosarcoma and melanoma tumors, and more recently on adipose tissue and skin as an organ (see $[2,3]$ and references within). Cell culture models include HL-60, Jurkat, and HCT116, the later including clones that are wildtype and null for p53.
However, a number of normal human leukocytes and a wide range of cancer cells have been tested. Cell responses have been measured for nsPEF effects on plasma membranes (integrity, potential, and phosphatidylserine externalization), endoplasmic reticulum (calcium mobilization), mitochondria (respiration, cytochrome $c$ release), and nucleus (fluorescence changes, DNA damage, roles for $\mathrm{p} 53$, and gene expression). We have also measured responses of adipose tissue, skin, and tumors. Studies with tumor tissues have determined responses from slowed tumor growth to tumor regression. These cell and tissue responses are distinct from response to classical plasma membrane electroporation.

A major question that remains to be fully investigated is the potential for nsPEF-induced cell-specific effects. There are two generalizations for cell-type-specific nsPEF-induced effects that have been defined. First, nsPEF-induced cell effects are not cell size-dependent as shown for classical plasma membrane electroporation where larger cells are more readily affected. While a wellcontrolled, extensive study has not been carried out for in vitro cell types, nsPEFs effects on the plasma membrane are more readily demonstrated in smaller cells compared to larger cells. Second, for a number of cell types tested, adherent cells have higher threshold for nsPEFinduced effects than cells that grow in suspension. Studies in progress are beginning to demonstrate selective effects in adipose tissue and skin.

It appears that nsPEFs can affect cells as a doubleedged sword; that is at relatively high electric fields nsPEFs recruit apoptosis mechanisms, but at lower electric fields they recruit nonapoptotic signaling mechanisms [2, 3]. It is now becoming clear that proteins that regulate apoptosis are also involved in regulating nonapoptotic processes. For example, we have shown that nsPEFs can modulate caspase activity and caspases have been shown to modulate apoptotic and nonapoptotic cell functions [2, 3] including proliferation, cell cycle, differentiation, as well as programmed cell death. So at higher electric fields nsPEFs can induce apoptosis resulting in cell death and size reduction and/or ablation of tumors. This is observed as direct electric field effects or biological responses to electric fields in the absence of drugs. This is distinct from electrochemotherapy where classical plasma membrane electroporation allows the entry of chemotherapeutic drugs such as bleomysin, which is toxic to the tumor.

At lower electric fields and shorter pulse durations, nsPEF recruit cell signaling mechanisms that induce calcium mobilization and modulate calcium-mediated functions [2, 3] such as platelet activation and aggregation, which is important for blood clotting. Activation of human platelets, Jurkat cells, and HL-60 cells mimic responses to hormones that act through G-protein-coupled, plasma membrane receptors that involve IP3 receptors in the endoplasmic reticulum. Furthermore, abrupt calcium mobilization has been shown to immobilize human neutrophils, presumably due to interruption of signals that direct specialized and specific mobilization in response to 
chemotactic signals. The mechanism(s) for these calcium mobilization responses remain to be determined. Nevertheless, since calcium is an ubiquitous second messenger signal, nsPEF-induced calcium modulation could have a wide range of applications.

We hypothesized that if nsPEFs affected the nuclear membrane, plasmids and transfected genes may enter the nucleus more readily and gene transcription may be enhanced. We demonstrated that when a green fluorescent protein (GFP) expression plasmid was transfected into cells with a classical plasma membrane electroporation pulse and then followed by a nonapoptotic nsPEF in a low-conductivity media, the level of expression and the number of cells expressing GFP were increased significantly $[2,3]$. The mechanism(s) for this result is not yet clear, but nsPEF-induced effects on the nucleus and DNA have been reported. While some studies suggest that direct electric field effects damage DNA, other studies indicate effect at the nucleus, some of which are reversible and nonlethal. Other studies indicate that nsPEF affect expression of endogenous genes. It remains to be determined if effects on transcription are due to actions on the nuclear membrane, gene transcription mechanisms, or both.

While these observations are exciting, only a few groups have carried out experiments with nsPEFs, but the numbers are growing. This is because generating pulses with such short durations, rapid rise times, and high electric fields is not a common skill. Funding from the Department of Defense through the Air Force Office of Scientific Research by a Multi-University Research Initiative has enhanced the growing number of studies using this new technology. These include investigators at Old Dominion University, Norfolk, where the technology originated and the MURI is administrated; Eastern Virginia Medical School, Norfolk; Harvard/MIT, Cambridge; Washington University, St Louis; University of Texas Health Science Center, San Antonio; Wisconsin University, Madison; Purdue University, Calumet; and Northwestern University, Evanston. A group at University of Southern California with Martin Gundersen and Tom Vernier, also funded by AFOSR, has also been productive with this technology. Furthermore, two Centers of Excellence Programs have been funded in Japan to include investigations of nsPEF effects on biological cells. In addition, groups in England, France, and Germany have begun to establish programs related to bioelectrics research, seeking help from the Center of Bioelectrics in Norfolk. Furthermore, all of the funded groups are training students, some of whom will continue studies in bioelectrics. Moreover, an undergraduate/graduate course in Bioelectrics is now offered at Old Dominion University and a wider range of bioelectrics-related courses may be offered in the future. Thus, it is likely that as work continues in this field, it will expand to other groups with other methodologies and expertise, and enhance our understanding of mechanisms that cells use to respond to unique nsPEF stimuli.
So!! Where do we go from here? Our initial strategies were to do preliminary studies to investigate a number of hypotheses based on understandings from classical plasma membrane electroporation. This approach revealed that a number of projects were worthy of pursuit based on peer-review publications and a wealth of unpublished data. However, a "rich kid in the candy store" strategy will not be successful. First, we are not rich. This technology requires a funding stream that is not easily acquired, especially with a new technology that must build a respected foundation in the peer-review processes. Thus, personnel and resources are limited. Second, acquisition of funding from national foundations such as the NIH, among others, requires focus in areas that are well defined, supported by unshakable preliminary data, and assured of success. Some funding may be available from venture capitol groups and startup companies, but the risk/benefit ratio must be favorable and this is not immediate with a new technology. Therefore, we have developed a strategy that is based on available funds, resources, and personnel to carry forth a series of studies that will provide for the future of bioelectrics. Bioelectrics research will remain stimulating because there is plenty of intracellular territory to explore.

Stephen J. Beebe

Karl H. Schoenbach

\section{REFERENCES}

[1] Kolb JF, Kono S, and Schoenbach KH. Nanosecond pulsed electric field generators for the study of subcellular effects. Bioelectromagnetics. In press.

[2] Schoenbach KH, Joshi RP, Kolb JF, et al. Ultrashort electrical pulses open a new gateway into biological cells. Proc. IEEE. 2004;92:1122-1137.

[3] Beebe SJ, White J, Blackmore PF, Deng Y, Somers K, and Schoenbach KH. Diverse effects of nanosecond pulsed electric fields on cells and tissues. DNA Cell Biol.2003;22(12):785-796.

[4] Hu Q, Viswanadham S, Joshi RP, Schoenbach KH, Beebe SJ, and Blackmore PF. Simulations of transient membrane behavior in cells subjected to a highintensity ultrashort electric pulse. Phys Rev E Stat Nonlin Soft Matter Phys. 2005;71(3 pt 1):031914.

Stephen J. Beebe received a BS in Zoology from Ohio University, Athens, 1970, and a $\mathrm{PhD}, 1982$, in medical sciences (pharmacology and biochemistry) from the Medical College of Ohio, Toledo. He did postdoctoral studies (1982-1987) in the Department of Molecular Physiology and Biophysics at the Howard Hughes Medical Institute, Vanderbilt, Nashville, Tenn, before serving as a Fulbright Scholar and Norwegian Marshall Scholar in Institute for Medical Biochemistry 
and the National Hospital in Oslo Norway (1987-1988). He is currently an Associate Professor at the Center for Pediatric Research, Departments of Pediatrics and Physiological Sciences, Eastern Virginia Medical School, Norfolk, Va. His research has focused on signal transduction mechanisms and structure-function studies of protein kinases using multiple disciplinary approaches utilizing pharmacology, biochemistry, physiology, molecular and cellular biology, and physics. Recently, he has helped develop the potential use of high-intensity nanosecond pulsed electric fields as a novel stimulus to regulate intracellular signal transduction. Dr. Beebe has received various honors including Outstanding Senior Visiting Scientist from the Norwegian Cancer Society while in Bergen Norway (1997-1999), the Iwao Yasuda Award by the Society for Physical Regulation in Biology and Medicine, and the Martin Black Award from the Institute of Physics and Engineering in Medicine.

Karl H. Schoenbach received the Diploma degree in physics and the Dr.rer.nat. degree in physics in 1966 and 1970, respectively, from the Technische Hochschule Darmstadt (THD), Germany. In 1985, he joined Old Dominion University in Norfolk, Va. He worked on photoconductive switches until 1993, but now he concentrates his research on high-pressure glow discharges, in

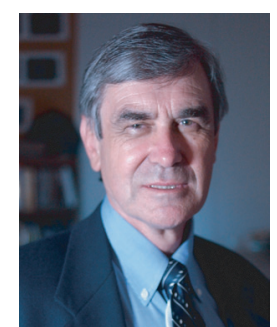
liquids, and environmental and medical ap-

plications of pulse power technology. He is currently the Director of the Center for Bioelectrics at Old Dominion University, and holds the Batten Chair of Bioelectric Engineering. Professor Schoenbach has chaired many workshops and conferences, including the First International Symposium on "Nonthermal Medical/Biological Treatments Using Electromagnetic Fields and Ionized Gases" in 1999. He was elected Fellow of IEEE in 1994 and received the 2000 High Voltage Award sponsored by IEEE-DEIS. He was Guest Editor of the IEEE Transactions on Electron-Devices (1990), the IEEE Transactions on Plasma Science (1999), the IEEE Transactions on Dielectrics and Electrical Insulation (2003), Coeditor of a book, "Low Temperature Plasma Physics" (2001), and is presently Coeditor of a book, "Atmospheric Pressure Air Plasmas," and an Associate Editor of the IEEE Transactions on Plasma Science. 

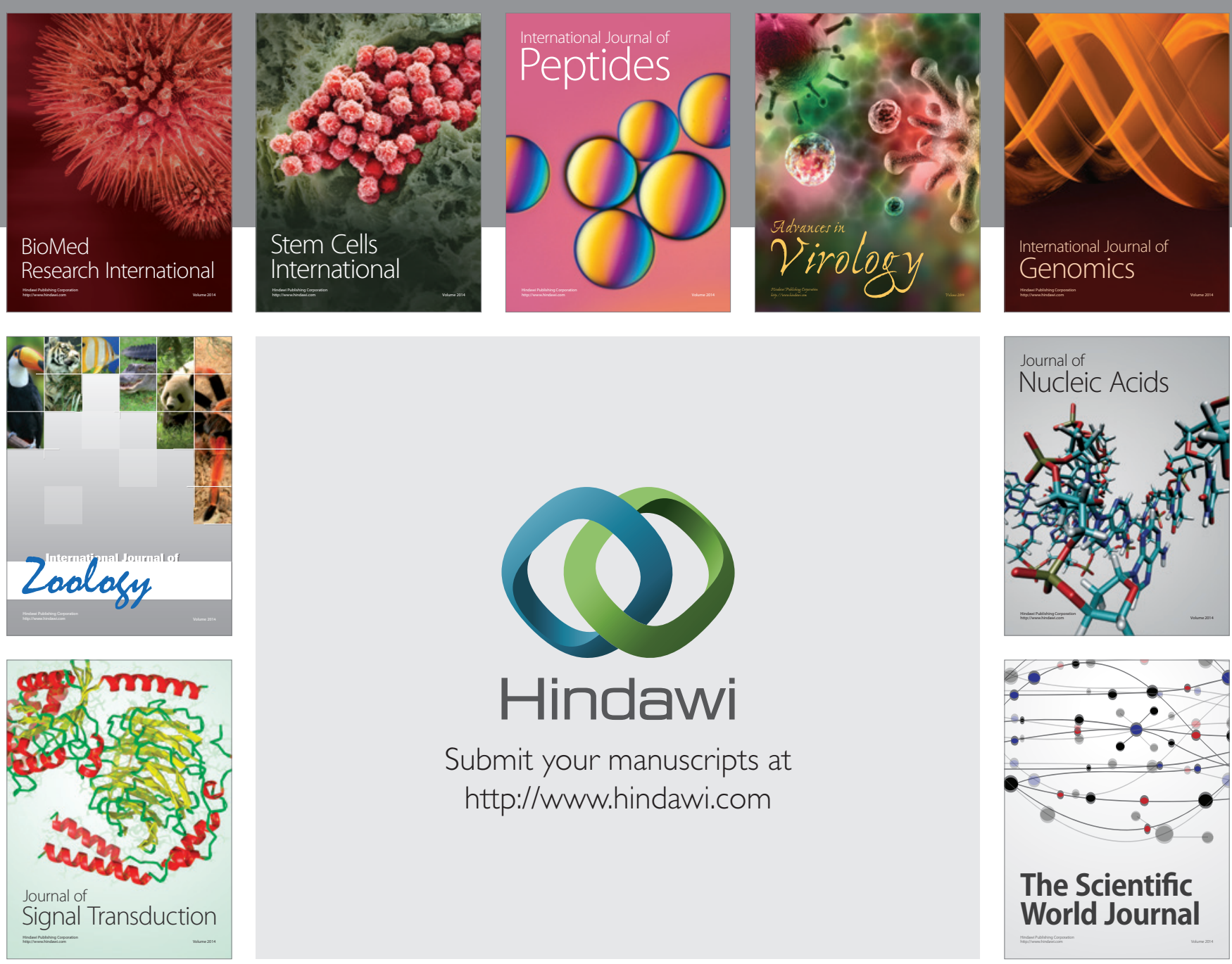

Submit your manuscripts at

http://www.hindawi.com
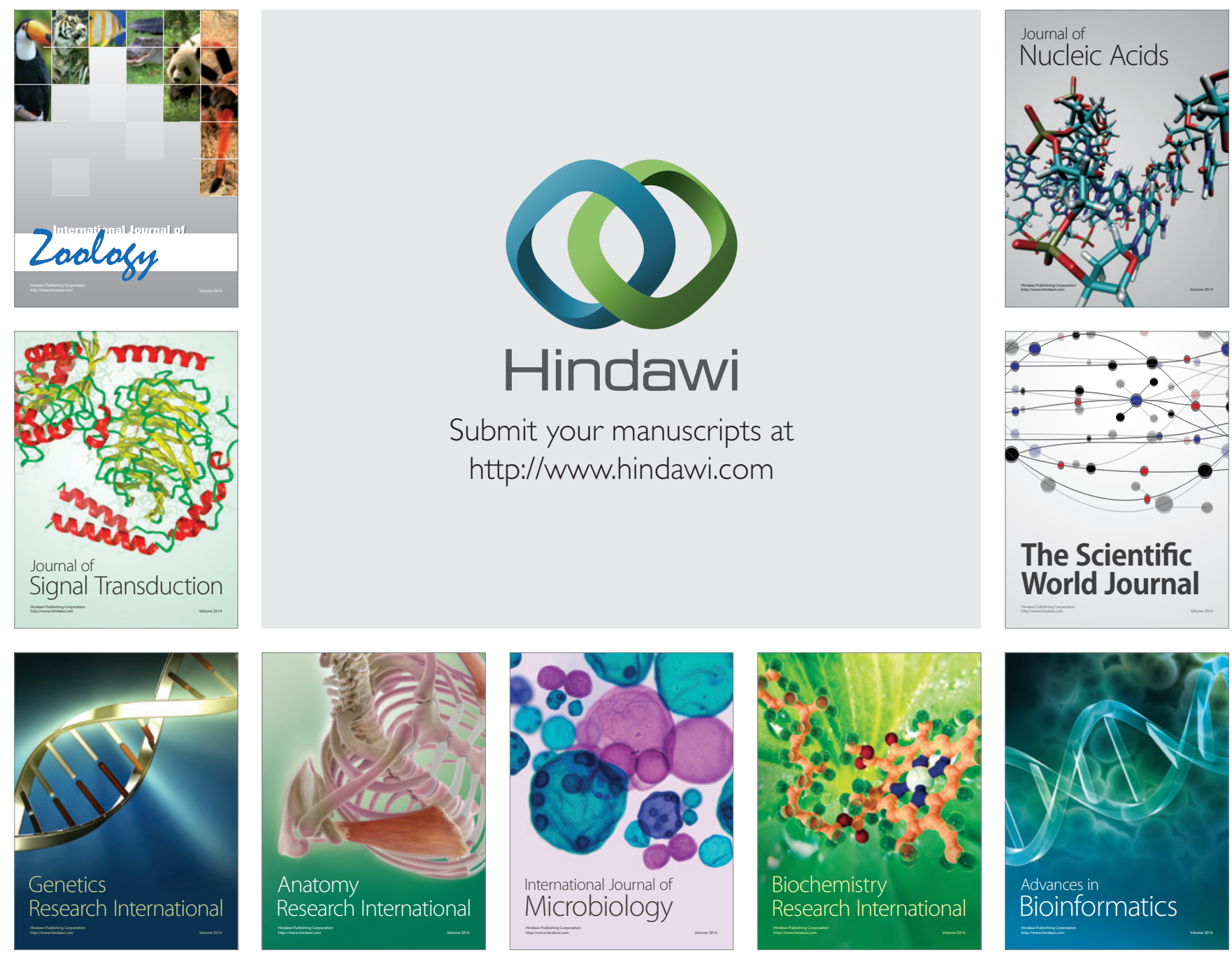

The Scientific World Journal
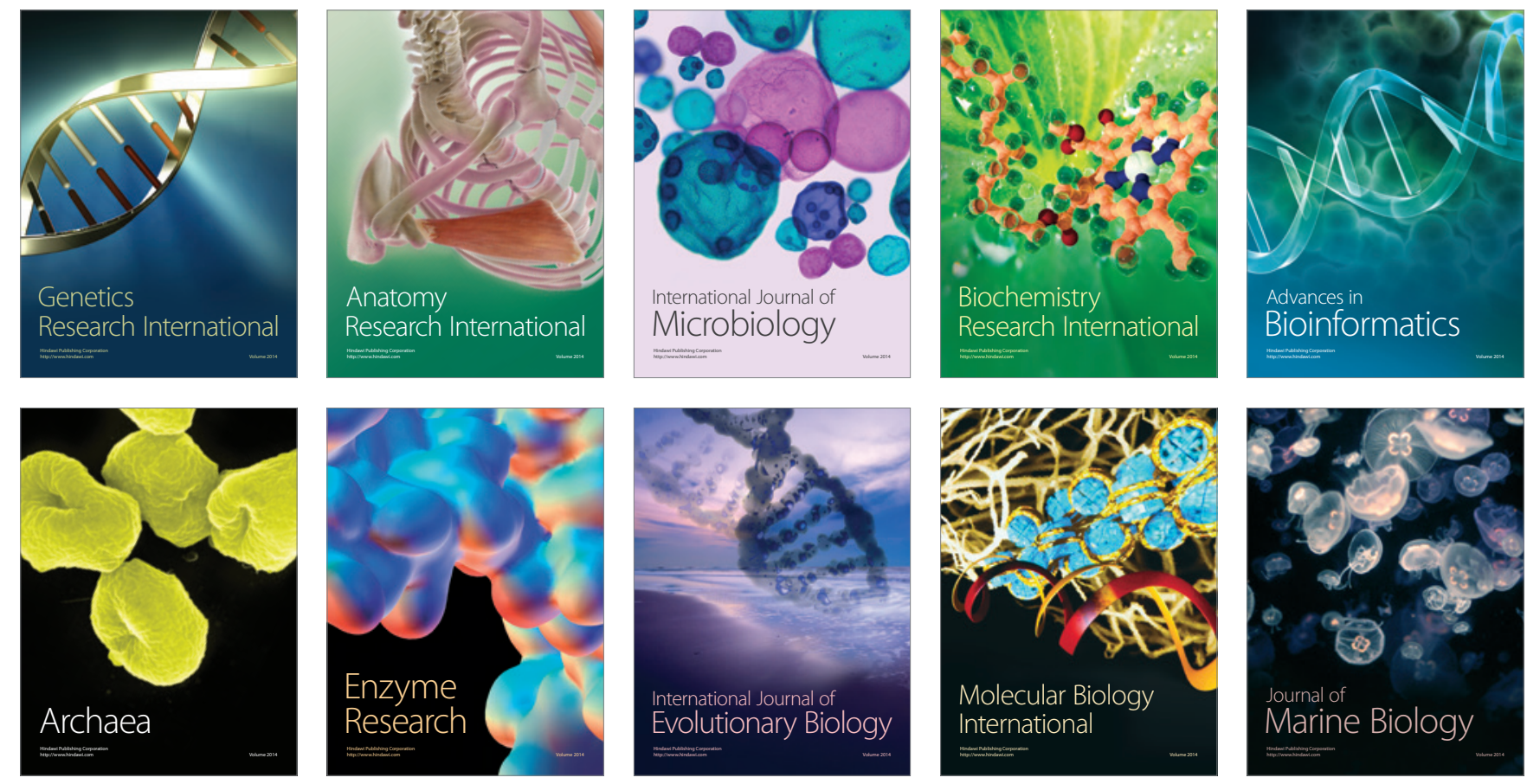